\title{
Pazopanib exposure decreases as a result of an ifosfamide-dependent drug-drug interaction: results of a phase I study
}

\author{
P Hamberg ${ }^{*}, 1,4$, M J Boers-Sonderen ${ }^{2}$, W T A van der Graaf ${ }^{2}$, P de Bruijn ${ }^{1}$, A B Suttle ${ }^{3}$, F A L M Eskens ${ }^{1}$, \\ $J$ Verweij ${ }^{1}, C M L$ van Herpen ${ }^{2}$ and S Sleijfer ${ }^{1}$ \\ ${ }^{1}$ Department of Medical Oncology and Cancer Genomics Netherlands, Erasmus MC Cancer Institute, Daniel den Hoed Cancer \\ Center, Rotterdam, The Netherlands; ${ }^{2}$ Radboud University Nijmegen Medical Center, Nijmegen, The Netherlands and \\ ${ }^{3}$ GlaxoSmithKline, Clinical Pharmacology Modeling and simulation, Research Triangle Park, NC, USA
}

Background: The vascular endothelial growth factor receptor (VEGFR) pathway plays a pivotal role in solid malignancies and is probably involved in chemotherapy resistance. Pazopanib, inhibitor of, among other receptors, VEGFR1-3, has activity as single agent and is attractive to enhance anti-tumour activity of chemotherapy. We conducted a dose-finding and pharmacokinetic (PK)/ pharmacodynamics study of pazopanib combined with two different schedules of ifosfamide.

Methods: In a $3+3+3$ design, patients with advanced solid tumours received escalating doses of oral pazopanib combined with ifosfamide either given 3 days continuously or given 3 -h bolus infusion daily for 3 days $\left(9 \mathrm{~g} \mathrm{~m}^{-2}\right.$ per cycle, every 3 weeks). Pharmacokinetic data of ifosfamide and pazopanib were obtained. Plasma levels of placental-derived growth factor (PIGF), vascular endothelial growth factor-A (VEGF-A), soluble VEGFR2 (sVEGFR2) and circulating endothelial cells were monitored as biomarkers.

Results: Sixty-one patients were included. Pazopanib with continuous ifosfamide infusion appeared to be safe up to $1000 \mathrm{mg}$ per day, while combination with bolus infusion ifosfamide turned out to be too toxic based on a variety of adverse events. Ifosfamidedependent decline in pazopanib exposure was observed. Increases in PIGF and VEGF-A with concurrent decline in sVEGFR2 levels, consistent with pazopanib-mediated VEGFR2 inhibition, were observed after addition of ifosfamide.

Conclusion: Continuous as opposed to bolus infusion ifosfamide can safely be combined with pazopanib. Ifosfamide co-administration results in lower exposure to pazopanib, not hindering biological effects of pazopanib. Recommended dose of pazopanib for further studies combined with 3 days continuous ifosfamide $\left(9 \mathrm{~g} \mathrm{~m}^{-2}\right.$ per cycle, every 3 weeks) is $800 \mathrm{mg}$ daily.

Given the heterogeneity of cancer, it is conceivable that combinations of anti-tumour agents render the best outcomes for patients with advanced solid malignancies.

The combination of conventional cytotoxic therapy with inhibition of the vascular endothelial growth factor receptor (VEGFR) pathway is attractive for several reasons. The VEGFR inhibition reduces the interstitial pressure of tumours rendering higher intratumoural levels of concomitantly administered cytotoxic agents and enhances effects of several cytotoxic agents at the tumour cell level (Boucher and Jain, 1992; Heldin et al, 2004; Willett et al, 2004). In addition, most VEGFR-inhibiting agents have no overlapping toxicities with conventional cytotoxics.

Pazopanib is a tyrosine kinase inhibitor targeting the VEGFR1-3, the platelet-derived growth factor receptors $\alpha$ and $\beta$ and c-kit

\footnotetext{
*Correspondence: Dr P Hamberg; E-mail: p.hamberg@sfg.nl

This study has been presented in part at the 2012 Annual meeting of the American Society of Clinical Oncology.

${ }^{4}$ Current address: Sint Franciscus Gasthuis, Rotterdam, The Netherlands.
} 
(Hamberg et al, 2010a). It received marketing approval for patients with metastatic renal cell carcinoma and for patients with advanced non-adipocytic soft tissue sarcomas (Sternberg et al, 2010; Van der Graaf et al, 2012). The recommended dose of pazopanib is $800 \mathrm{mg}$ once daily with fatigue, diarrhoea, nausea, hypertension and elevated liver-enzymes as most common toxicities (Van der Graaf et al, 2012).

Ifosfamide is standard of care for different tumour types including advanced soft tissue sarcomas, where a continuous and bolus infusion schedule have shown equivalent activity (Lorigan et al, 2007). Myelosuppression, febrile neutropenia (FN) and encephalopathy are the most relevant toxicities (Lorigan et al, 2007).

Given the potential of the combination of pazopanib and ifosfamide, we performed a phase I study to determine the recommended dose of pazopanib combined with ifosfamide in two different schedules.

\section{PATIENT AND METHODS}

Patient selection. Patients with advanced or metastatic solid tumours for whom ifosfamide-based therapy was considered appropriate or for whom no standard therapy was available were eligible. Other inclusion criteria included: ECOG performance status $<2$, evaluable or measurable disease (RECIST 1.1) (Eisenhauer et al, 2009), age $\geqslant 18$ years, adequate bone marrow, liver, and renal function, and systolic blood pressure (BP) $<160 \mathrm{~mm} \mathrm{Hg}$ and diastolic BP $<90 \mathrm{~mm} \mathrm{Hg}$ (two antihypertensive drugs allowed). Main exclusion criteria were: history of cardiovascular disease other than hypertension and signs/symptoms of central nervous system metastases.

The study was approved by the institutional review boards and conducted in accordance with the principles embodied in the Declaration of Helsinki. Written informed consent was obtained from each participant prior to enrollment.

Study design. Daily oral pazopanib was evaluated in combination with a fixed dose of ifosfamide $9 \mathrm{~g} \mathrm{~m}^{-2}$ per cycle, either given as 3 days continuous intravenous infusion (CIV) or as $3 \mathrm{~h}$ bolus intravenous infusion (BIV) for 3 consecutive days, both at 3-weekly intervals. Pazopanib was escalated in serial cohorts at a dose of 400, 800 and $1000 \mathrm{mg}$ daily. If the maximal tolerated dose (MTD) would be exceeded at $400 \mathrm{mg}$ then an extra cohort exploring pazopanib at $200 \mathrm{mg}$ daily was added.

The $3+3+3$ design, a novel model recently proposed aiming to reduce falsely halting dose escalation in combination phase I trials, was applied in the original protocol (Hamberg et al, 2010b). If a dose-limiting toxicity (DLT) was observed in one patient, three additional patients were recruited at that dose level, with dose escalation proceeding if no further DLT occurred at that dose level. If DLT was observed in two out of six, three additional patients were enrolled. If a DLT occurred in 2 out of $3,>2$ out of 6 or $>2$ out of 9 patients in a cohort, MTD had been exceeded. The MTD was defined as the highest dose level with a DLT incidence of $<33 \%$.

In order to be exposed to steady-state concentrations of pazopanib and to determine the effects of ifosfamide administration on pazopanib pharmacokinetics (PK), patients in the dose-escalation phase started on pazopanib 7 days prior to the first cycle of ifosfamide. At the MTD, six additional patients were treated in an expansion cohort to get better insight into the safety profile, to confirm the MTD, and to further study the PK interaction. For the latter, pazopanib was started 7 days after the first ifosfamide cycle in the patients in the expansion phase, which enabled an intra-patient comparison of ifosfamide PK with or without the presence of pazopanib (Figure 1).

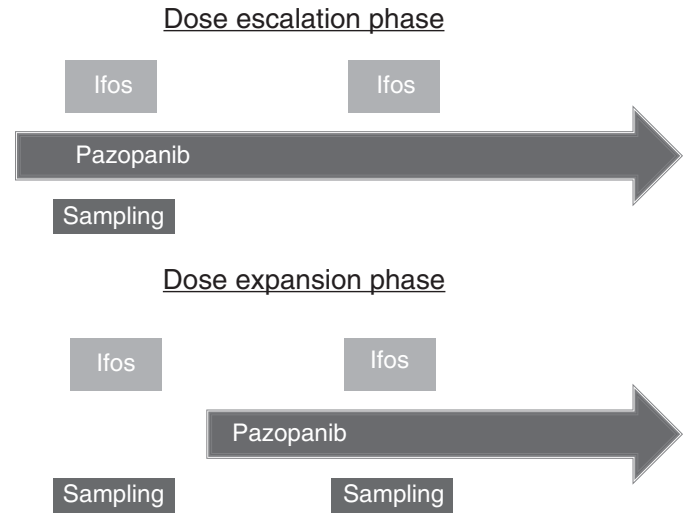

Figure 1. Study design. Abbreviation: Ifos = ifosfamide.

Using the Common Terminology Criteria for adverse events, version 3.0, DLT during the first treatment cycle (in the dose expansion phase during the first two treatment cycles) was defined as: grade 4 neutropenia $\geqslant 7$ days, FN, grade 4 thrombocytopenia, creatinine clearance $\leqslant 50 \mathrm{ml} \mathrm{min}^{-1}$, grade 3-4 proteinuria or any drug-related grade 3 or 4 non-haematological toxicity. Hypertension was considered DLT in case of symptomatic hypertension; persistent $(>24 \mathrm{~h})$ and asymptomatic systolic BP $>170 \mathrm{~mm} \mathrm{Hg}$ and/or diastolic BP >100 $\mathrm{mm} \mathrm{Hg}$; systolic BP 160-170 and/or diastolic BP 90-100 that could not be controlled within 2 weeks; or an increase of diastolic BP $>20 \mathrm{~mm} \mathrm{Hg}$, which despite antihypertensive medication was not adequately controlled within 2 weeks. A dose delay or interruption exceeding 2 weeks was classified as DLT. If neutropenia comprised the predominant DLT at a certain dose level, that and subsequent levels were explored in combination with granulocyte cell stimulating factor (pegfilgrastim $6 \mathrm{mg}$ once per cycle).

Patients were treated for a maximum of six ifosfamide cycles. Patients experiencing clinical benefit from the combination of pazopanib and ifosfamide were allowed to continue treatment thereafter with pazopanib until disease progression or unacceptable toxicity.

PK sampling and analysis. Concentrations of ifosfamide and its most important metabolites, 2-dechloroethyl-ifosfamide, 3-dechloroethyl-ifosfamide and 4-hydroxy-ifosfamide were quantitated as previously reported in 11-16 samples per ifosfamide cycle (Hamberg et al, 2010c).

For the analysis of pazopanib, 15 samples per patient were drawn and quantitated as previously reported (Hurwitz et al, 2009).

Statistical data analysis. Plasma concentrations of ifosfamide and its metabolites were plotted as a function of time. Area under the curve (AUC) was calculated by the trapezoidal method. Noncompartmental PK analysis including half-life $\left(T^{1 / 2}, \mathrm{~h}\right)$ was calculated using the software package WinNonlin version 6.1. Total body clearance of ifosfamide was calculated by dividing the administered dose by the AUC of ifosfamide. Statistical analysis were made using the software package SPSS (v20). Correlation of the changes in AUC or clearance and half-life was evaluated by a two-sided paired $t$-test for subjects in the expansion cohorts.

Median plasma pazopanib concentration-time profiles were generated for subjects in the dose escalation cohorts. The area under the plasma pazopanib concentration-time curve from 0 to $24 \mathrm{~h}$ (AUC (0-24)) was calculated using nominal blood sample collection times after administration of pazopanib on day 21 cycle 1 (pazopanib alone) and day 3 cycle 2 (pazopanib plus ifosfamide) for subjects in the expansion cohorts. 
Biomarker analysis. Biomarker samples were collected in all patients at baseline and prior cycle 2 day 1 . During the escalation phase, an additional sample was drawn prior to day 1 cycle 1 and during the expansion prior to the first pazopanib dose. Circulating endothelial cell (CEC) enumeration was determined with a flow-cytometry-based method (Kraan et al, 2012). Plasma concentrations of VEGF-A, soluble VEGFR2 (sVEGFR2) and placental-derived growth factor (PlGF) were determined using ELISA (R\&D Systems, Minneapolis, MN, USA) according to the manufacturers' instructions.

\section{RESULTS}

Dose escalation, MTD and dose intensity. In total, 61 patients were enrolled (Table 1), 29 in the CIV schedule and 32 on the BIV schedule. In all, 15 out of 61 patients were not evaluable for determination of DLT of the combination and were replaced. The most common reason was early progression $(n=5)$. Two other patients were registered but did not receive a dose of study drug at all. Three patients appeared not to tolerate the single agent treatment they received before the second agent was added. As the aim of this study was to identify the MTD of the combination of pazopanib and ifosfamide, it was decided to replace these patients. Five others were not evaluable due to a diversity of reasons including withdrawal of consent (not based on toxicity), and an allergic reaction to mesna, which was prophylactically administered with ifosfamide.

In the CIV arm, no DLTs were observed in the three evaluable patients at the first dose level, whereas 1 DLT (FN) was observed at a dose level with $800 \mathrm{mg}$ pazopanib (six evaluable patients). At the highest pre-defined dose level of $1000 \mathrm{mg}$ pazopanib two DLTs

\begin{tabular}{|l|c|}
\hline \multicolumn{2}{|l|}{ Table 1. Demographics and baseline characteristics } \\
\hline Age (median, years) & 56 (range 18-76) \\
\hline Gender & $40(65)$ \\
\hline Male & $21(35)$ \\
\hline Female & \\
\hline WHO & $23(38)$ \\
\hline 0 & $38(62)$ \\
1 & \\
\hline Tumour type & 19 \\
\hline Sarcoma (incl GIST) & 6 \\
Melanoma & 6 \\
Urothelial carcinoma & 5 \\
Ovarian carcinoma & 5 \\
Carcinoma of unknown primary & 4 \\
Prostate & 3 \\
Gastric carcinoma & 3 \\
Oesophageal carcinoma & 10 \\
Miscellaneous & \\
&
\end{tabular}

Previous non-hormonal systemic anticancer treatment

\begin{tabular}{|l|r|}
\hline 0 & 12 \\
1 & 29 \\
2 & 11 \\
4 & 3 \\
5 & 4 \\
\hline Abbreviation: GIST = gastro-intestinal stromal tumour. \\
\hline
\end{tabular}

occurred (FN and encephalopathy) in nine patients. During expansion phase $(n=6)$ at this dose level, no additional DLTs were observed. At the MTD in the CIV arm, the dose intensity of ifosfamide and pazopanib was $92 \%$ and $93 \%$, respectively, with a median number of ifosfamide cycles of 4 .

In the first two patients treated with $400 \mathrm{mg}$ pazopanib in the BIV arm, an episode of FN was encountered. Adding G-CSF to the $400 \mathrm{mg}$ pazopanib dose level three DLTs were observed in nine patients (grade 3 encephalopathy, grade 3 proteinuria and grade 3 pneumonia during neutropenia). The dose of pazopanib was deescalated to $200 \mathrm{mg}$ and supported with G-CSF. In two out of the first nine evaluable patients DLTs occurred (one case each of grade 3 encephalopathy and FN). The subsequent dose expansion in another six patients resulted in three more DLTs (pneumonia during neutropenia and grade 5 cardiac arrest in one patient, renal toxicity and grade 3 fatigue).

Toxicity. In addition to hematological toxicity, the main grades 3-4 toxicities during combination therapy were fatigue and hypophosphatemia, irrespective of treatment schedule. Grade 3-4 vomiting was more pronounced in the BIV-treated patients, whereas all grades of hypertension and grade $3 / 4$ neutropenia occurred more often in the CIV group. (Table 2)

Pharmacokinetics. Pazopanib had no impact on plasma half-life, AUC or clearance, of ifosfamide or any of its metabolites. Figures 2 and 3 depicts the ifosfamide concentrations with and without concomitant administration of pazopanib.

In contrast, pazopanib concentrations declined by $\sim 35 \%$ within $72 \mathrm{~h}$ during concomitant ifosfamide infusion in patients in the dose escalation phase during CIV (Figure 4). There seems to be a time-dependent effect, resulting in comparable median plasma pazopanib concentrations at the end of ifosfamide infusion across dose-levels of pazopanib (Figure 4).

$\mathrm{PK}$ analysis in patients treated in the dose expansion phase revealed that the mean AUC (0-24) of pazopanib was reduced by $\sim 27 \%$ upon co-administration of ifosfamide as compared with the AUC of pazopanib single agent (Figure 5).

Biomarker analysis. During the treatment with pazopanib, there was a dose-dependent increase in PlGF and VEGF-A with a concurrent decline in sVEGFR2 (Table 3). Importantly, this phenomenon remained intact after the addition of ifosfamide. No consistent pattern was seen by enumeration of CEC (data not shown).

Anti-tumour activity. Of 45 patients evaluable for response, 10 partial responses were observed: 4 in the CIV-treated patients ( 2 patients with synovial sarcoma, 1 each with ovarian and prostate cancer) and 6 patients in the BIV schedule (urothelial cancer $(n=2)$, one each with sarcoma not otherwise specified, mesothelioma, ovarian cancer and an cancer of unknown primary). Prolonged disease stabilisation defined as stable disease for at least 3 months was noted in nine and five patients in the CIV and BIV group, respectively.

\section{DISCUSSION}

In this study, we explored safety and tolerability of the combination of pazopanib and ifosfamide, and its possible dependence on the ifosfamide infusion schedule. Pazopanib in combination with BIV-administered ifosfamide turned out to be intolerable. In contrast, if ifosfamide is continuously administered over 3 days, pazopanib could be escalated to dose levels exceeding the registered single agent dose of $800 \mathrm{mg}$ daily. The inclusion of a dose higher than the registered single agent dose was done, since in a previous study a drug-drug interaction between ifosfamide and sunitinib resulted in a decreased exposure to sunitinib (Hamberg 
Table 2. Most frequent adverse events during the first and during all combination cycles

\begin{tabular}{|c|c|c|c|c|c|c|c|c|}
\hline & \multicolumn{4}{|c|}{$\mathrm{CIV}(n=26)$} & \multicolumn{4}{|c|}{$\operatorname{BIV}(n=27)$} \\
\hline & \multicolumn{2}{|c|}{$\begin{array}{c}\text { First cycle adverse } \\
\text { events }\end{array}$} & \multicolumn{2}{|c|}{$\begin{array}{l}\text { Adverse events at all } \\
\text { combination cycles }\end{array}$} & \multicolumn{2}{|c|}{$\begin{array}{c}\text { First cycle adverse } \\
\text { events }\end{array}$} & \multicolumn{2}{|c|}{$\begin{array}{l}\text { Adverse events at all } \\
\text { combination cycles }\end{array}$} \\
\hline & Grade 3/4 & All grades & Grade 3/4 & All grades & Grade 3/4 & All grades & Grade $3 / 4$ & All grades \\
\hline Anaemia & 1 & 21 & 5 & 26 & 0 & 23 & 4 & 27 \\
\hline Thrombocytopaenia & 1 & 7 & 3 & 13 & 3 & 14 & 5 & 17 \\
\hline Neutropaenia & 23 & 24 & 25 & 25 & 13 & 16 & 15 & 19 \\
\hline Fatigue & 2 & 24 & 6 & 25 & 2 & 20 & 5 & 23 \\
\hline Nausea & 1 & 24 & 2 & 25 & 2 & 22 & 2 & 23 \\
\hline Vomiting & 0 & 15 & 0 & 21 & 5 & 20 & 6 & 21 \\
\hline Mucositis & 0 & 8 & 0 & 11 & 0 & 4 & 0 & 8 \\
\hline Anorexia & 0 & 17 & 0 & 22 & 1 & 15 & 4 & 18 \\
\hline Constipation & 0 & 17 & 0 & 19 & 0 & 13 & 0 & 15 \\
\hline Diarrhoea & 0 & 10 & 0 & 15 & 1 & 7 & 1 & 11 \\
\hline Hypertension & 1 & 10 & 2 & 13 & 1 & 3 & 2 & 4 \\
\hline Encepahlopathy & 1 & 10 & 1 & 14 & 2 & 9 & 3 & 14 \\
\hline ALAT & 0 & 13 & 0 & 19 & 0 & 11 & 1 & 12 \\
\hline ASAT & 0 & 14 & 1 & 19 & 0 & 12 & 0 & 16 \\
\hline Hypophosphatemia & 2 & 11 & 5 & 13 & 1 & 12 & 5 & 17 \\
\hline Proteinuria & 1 & 11 & 1 & 12 & 0 & 9 & 0 & 15 \\
\hline
\end{tabular}

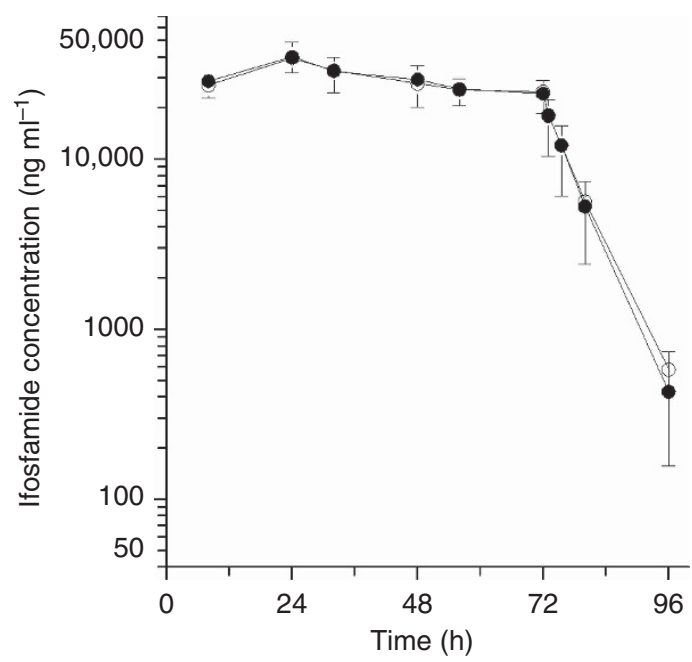

Figure 2. Mean concentrations (plus s.d.) of ifosfamide administered alone (closed symbols: bars up) or in combination with pazopanib (open symbols, bars down) during the 3 days continuous infusions.

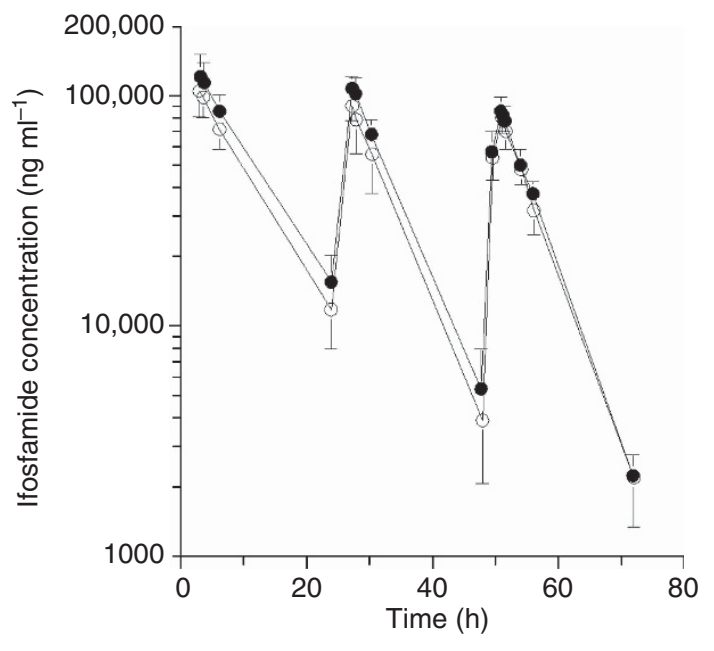

Figure 3. Mean concentrations (plus s.d.) of ifosfamide administered alone (closed symbols, bars up) or in combination with pazopanib (open symbols, bars down) during 3 consecutive bolus infusion days. et al, 2010c). This observation was confirmed in the current study, showing a PK interaction between pazopanib and ifosfamide.

Our study clearly underlines that the administration schedule of one of the drugs of a combination can have a major impact on the tolerability of the combination. One of the reasons that could account for the observed differences in tolerability between the two schedules of ifosfamide might be a schedule-dependent drugdrug interaction. Previously, pazopanib has demonstrated PK interactions with conventional chemotherapy, resulting in higher exposure to paclitaxel or the combination of paclitaxel and carboplatin (Tan et al, 2010; Burris et al, 2012; Du Bois et $a$, 2012). In contrast, combining pazopanib with full-dose gemcitabine was feasible up to $800 \mathrm{mg}$ pazopanib and no PK

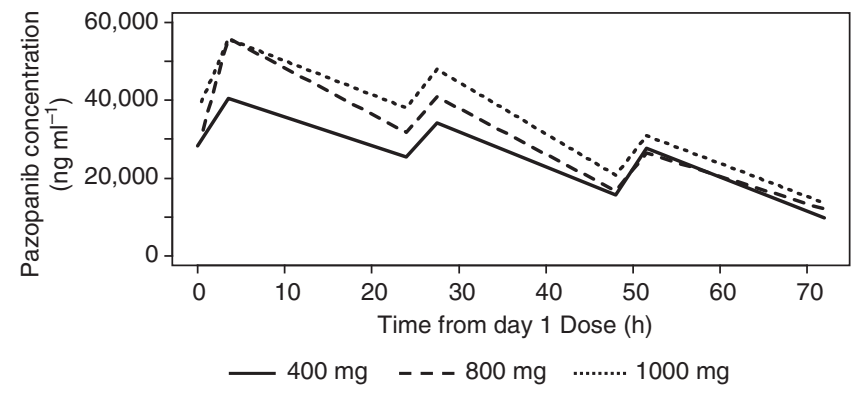

Figure 4. Median plasma pazopanib concentration time profile (dose escalation, in patients with continuously given ifosfamide). 
interaction was found (Plummer et al, 2013). Currently, extensive PK analysis did not show an impact of pazopanib on serum levels of ifosfamide or its metabolites.

In contrast, pazopanib exposure was clearly lowered by concurrent infusion of ifosfamide. This effect of ifosfamide on pazopanib levels yielded in similar pazopanib concentrations at the end of the ifosfamide infusion, regardless of the dose of pazopanib given. A potential underlying mechanism might be ifosfamide's inductive effects on CYP3A (Boddy et al, 1995) for which pazopanib, in addition to being a weak inhibitor, is also a substrate (Goh et al, 2010). If the induction of CYP3A by ifosfamide is indeed the cause of the decreased pazopanib exposure, this could imply that other drugs able to induce CYP3A should be avoided during pazopanib therapy but this warrants further exploration.

Yet, this PK interaction does not explain the striking difference in tolerability between the CIV and BIV schedule. In a study in patients with soft tissue sarcoma, BIV and CIV administrations of ifosfamide were compared demonstrating CIV to be slightly less toxic in terms of a lower rate of treatment discontinuation due to toxicity and a lower incidence of grade 3-4 dyspnoe and infection. However, CIV induced a higher incidence of grade 3-4 anaemia and grade 2-3 nausea than BIV ifosfamide (Lorigan et al, 2007). The higher incidences of grade 3-4 neutropenia in the CIV schedule $(88 \%$; 23 out of 26 patients) compared with the BIV (48\%; 13 out of 27 patients) observed in this study were in contrast with the study by Lorigan et al (2007) in which comparable incidences in CIV- and BIV-treated patients for grade 3-4 neutropenia incidence $(62.7 \%$ vs $60.0 \%)$ were found. This strongly suggests that

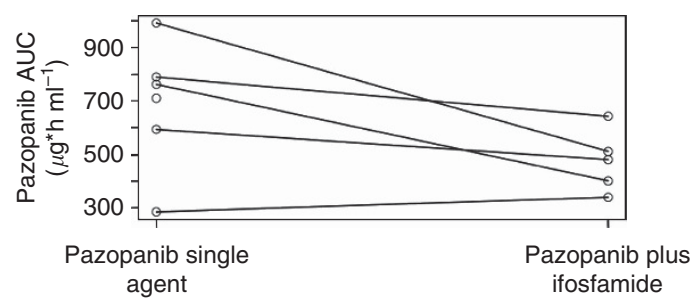

Figure 5. Pazopanib AUC administered as single agent (day 21, cycle 1) vs combined administration with continuous ifosfamide (day 3, cycle 2) in the dose expansion phase. the potentiating effect of pazopanib on the occurrence of neutropenia depends on the chosen infusion schedule. Autoinduction of ifosfamide is $52 \%$ higher during bolus infusion as compared with continuous infusion (Kerbusch et al, 2001) and as a result higher concentrations of ifosfamide are generated. Accordingly, the $\mathrm{AUC}_{0-72}$ of ifosfamide in our trial was indeed higher during bolus infusions as compared with the continuous infusion $\left(3149 \mu \mathrm{g}^{\star} \mathrm{h} \mathrm{ml}^{-1}\right.$ vs $2234 \mu \mathrm{g}^{\star} \mathrm{h} \mathrm{ml}^{-1}$, respectively).

Three cytokines known to reflect biological effects from VEGFR-TKIs showed alterations in PIGF, VEGF-A and sVEGFR levels following exposure to pazopanib consistent with inhibition of VEGFR2 activity (Sleijfer et al, 2012). Ifosfamide did not nullify these pazopanib-induced biological effects, so ifosfamide does not hinder pazopanib to exert biological effects despite its lowering effects on pazopanib-levels.

This study shows that the upper boundary of safe dosing is, at least, $1000 \mathrm{mg}$ per day pazopanib with CIV ifosfamide $9 \mathrm{~g} \mathrm{~m}^{-2}$. Like the single-agent pazopanib dose-finding study, the tolerable dose is higher than the currently approved dose, chosen on the basis of PK parameters and analyses of biological activity (Hurwitz et al, 2009). On the basis of the facts that the exposure to pazopanib during ifosfamide is comparable in patients using $1000 \mathrm{mg}$ per day and $800 \mathrm{mg}$ per day after $48 \mathrm{~h}$ (Figure 4) and that the pharmacodynamic parameters showed that the short period of lower pazopanib levels using $800 \mathrm{mg}$ compared with $1000 \mathrm{mg}$ pazopanib did not have any effects on the pazopanib-induced biological effects, both in line with findings from the phase I trial on pazopanib monotherapy (Hurwitz et al, 2009), we recommend as dose for further studies $800 \mathrm{mg}$ pazopanib and CIV ifosfamide $9 \mathrm{~g} \mathrm{~m}^{-2}$.

Importantly, this is the first phase I study to apply the $3+3+3$ design. This pre-planned design for combination phase I trials aims to eliminate chances of falsely halting dose escalation, based on a high a priori chance of developing a DLT of one of the drugs (Hamberg et al, 2010b). In the CIV arm, two out of the first six patients at the $1000 \mathrm{mg}$ pazopanib dose level experienced a DLT. According to the conventional $3+3$ design, this would have been interpreted as toxicity exceeding MTD. However, no DLTs were encountered in the other nine patients enrolled in this dose level. This strongly underscores the clinical applicability of this $3+3+3$-approach in establishing the tolerability of drug combinations.

Table 3. Biomarkers in the dose escalation cohorts (baseline normalised to 1)

\begin{tabular}{|c|c|c|c|c|c|}
\hline $\begin{array}{l}\text { Ifosfamide } \\
\text { schedule }\end{array}$ & Biomarker & Pazopanib dose & Baseline & $\begin{array}{l}\text { After } 7 \text { days pazopanib } \\
\text { but prior to first ifosfamide }\end{array}$ & $\begin{array}{l}\text { Prior to second } \\
\text { ifosfamide }\end{array}$ \\
\hline \multirow[t]{9}{*}{$\mathrm{CIV}$} & \multirow[t]{3}{*}{ PIGF } & 400 & 1 & 1.75 & 1.35 \\
\hline & & 800 & 1 & 6.87 & 8.46 \\
\hline & & 1000 & 1 & 7.13 & 4.39 \\
\hline & \multirow[t]{3}{*}{ VEGF-A } & 400 & 1 & 1.08 & 0.54 \\
\hline & & 800 & 1 & 5.26 & 5.72 \\
\hline & & 1000 & 1 & 3.85 & 2.73 \\
\hline & \multirow[t]{3}{*}{ sVEGFR2 } & 400 & 1 & 0.89 & 0.88 \\
\hline & & 800 & 1 & 0.77 & 0.70 \\
\hline & & 1000 & 1 & 0.76 & 0.69 \\
\hline \multirow[t]{6}{*}{ BIV } & \multirow[t]{2}{*}{ PIGF } & 200 & 1 & 1.81 & 1.75 \\
\hline & & 400 & 1 & 2.40 & 3.24 \\
\hline & \multirow[t]{2}{*}{ VEGF-A } & 200 & 1 & 1.72 & 3.12 \\
\hline & & 400 & 1 & 1.96 & 2.20 \\
\hline & \multirow[t]{2}{*}{ sVEGFR-2 } & 200 & 1 & 0.89 & 0.81 \\
\hline & & 400 & 1 & 0.79 & 0.77 \\
\hline
\end{tabular}


In conclusion, this study has clearly demonstrated that tolerability of pazopanib and ifosfamide is dependent on the infusion schedule. An evident explanation for the observed differences is not readily available. Furthermore, ifosfamide appeared to lower pazopanib levels, but despite the lower levels of pazopanib, it still exerted biological activity. In addition, this study stresses the importance of the $3+3+3$ design for exploring drug combinations in phase I studies when one of the agents is known to induce high rates of toxicity. Last, based upon our data the dose recommended for pazopanib when combined with CIV ifosfamide $9 \mathrm{~g} \mathrm{~m}^{-2}$ is $800 \mathrm{mg}$, while a combination with bolus ifosfamide is not feasible. Further studies on the combination of pazopanib and ifosfamide are currently being designed.

\section{ACKNOWLEDGEMENTS}

Role of the funding source: This investigator-initiated study was supported by GSK. The study was designed by the academic researchers. Trial registration number NTR 2063.

\section{CONFLICT OF INTEREST}

ABS is an employee of GSK (pazopanib). All remaining authors declare no conflict of interest.

\section{REFERENCES}

Boddy AV, Cole M, Pearson AD, Idle JR (1995) The kinetics of the autoinduction of ifosfamide metabolism during continuous infusion. Cancer Chemother Pharmacol 36: 53-60.

Boucher Y, Jain RK (1992) Microvascular pressure is the principal driving force for interstitial hypertension in solid tumors: implications for vascular collapse. Cancer Res 52: 5110-5114.

Burris 3rd HA, Dowlati A, Moss RA, Infante JR, Jones SF, Spigel DR, Levinson KT, Lindquist D, Gainer SD, Dar MM, Suttle AB, Ball HA, Tan AR (2012) Phase I study of pazopanib in combination with paclitaxel and carboplatin given every 21 days in patients with advanced solid tumors. Mol Cancer Ther 11: 1820-1828.

Du Bois A, Vergote I, Wimberger P, Ray-Coquard I, Harter P, Curtis LB, Mitrica I (2012) Open-label feasibility study of pazopanib, carboplatin, and paclitaxel in women with newly diagnosed, untreated, gynaecologic tumours: a phase I/II trial of the AGO study group. Br J Cancer 106: 629-632.

Eisenhauer EA, Therasse P, Bogaerts J, Schwartz LH, Sargent D, Ford R, Dancey J, Arbuck S, Gwyther S, Mooney M, Rubinstein L, Shankar L, Dodd L, Kaplan R, Lacombe D, Verweij J (2009) New response evaluation criteria in solid tumours: revised RECIST guideline (version 1.1). Eur J Cancer 45: 228-247.

Goh BC, Reddy NJ, Dandamudi UB, Laubscher KH, Peckham T, Hodge JP, Suttle AB, Arumugham T, Xu Y, Xu CF, Lager J, Dar MM, Lewis LD (2010) An evaluation of the drug interaction potential of pazopanib, an oral vascular endothelial growth factor receptor tyrosine kinase inhibitor, using a modified cooperstown $5+1$ cocktail in patients with advanced solid tumors. Clin Pharmacol Ther 88: 652-659.

Hamberg P, Verweij J, Sleijfer S (2010a) (Pre-)clinical pharmacology and activity of pazopanib, a novel multikinase angiogenesis inhibitor. The Oncologist 15: 539-547.

Hamberg P, Ratain MJ, Lesaffre E, Verweij J (2010b) Dose-escalation models for combination phase I trials in oncology. Eur J Cancer 46: 2870-2878.
Hamberg P, Steeghs N, Loos WJ, van de Biessen D, den Hollander M, Tascilar M, Verweij J, Gelderblom H, Sleijfer S (2010c) Decreased exposure to sunitinib due to concomitant administration of ifosfamide: results of a phase I and pharmacokinetic study on the combination of sunitinib and ifosfamide in patients with advanced solid malignancies. Br J Cancer 102: 1699-1706.

Heldin CH, Rubin K, Pietras K, Ostman A (2004) High interstitial fluid pressure - an obstacle in cancer therapy. Nat Rev Cancer 4: 806-813.

Hurwitz HI, Dowlati A, Saini S, Savage S, Suttle AB, Gibson DM, Hodge JP, Merkle EM, Pandite L (2009) Phase I trial of pazopanib in patients with advanced cancer. Clin Cancer Res 15: 4220-4227.

Kerbusch T, Mathot RAA, Keizer HJ, Kaijser GP, Schellens JH, Beijnen JH (2001) Influence of dose and infusion duration on pharmacokinetics of ifosfamide and metabolites. Drug Metab Dispos 29: 967-975.

Kraan J, Strijbos MH, Sieuwerts AM, Foekens JA, den Bakker MA, Verhoef C (2012) A new approach for rapid and reliable enumeration of circulating endothelial cells in patients. J Thromb Haemost 10: 931-939.

Lorigan P, Verweij J, Papai Z, Rodenhuis S, Le Cesne A, Leahy MG, Radford JA, Van Glabbeke MM, Kirkpatrick A, Hogendoorn PC, Blay JY, European Organisation for Research and Treatment of Cancer Soft Tissue and Bone Sarcoma Group Study (2007) Phase III trial of two investigational schedules of ifosfamide compared with standard-dose doxorubicin in advanced or metastatic soft tissue sarcoma: a European Organisation for Research and Treatment of Cancer soft tissue and bone sarcoma group study. J Clin Oncol 25: 3144-3150.

Plummer R, Madi A, Effels M, Richly H, Nokay B, Rubin S, Ball HA, Weller S, Botbyl J, Gibson DM, Scheulen ME (2013) A phase I study of pazopanib in combination with gemcitabine in patients with advanced solid tumors. Cancer Chemother Pharmacol 71: 93-101.

Sleijfer S, Gorlia T, Lamers C, Burger H, Blay JY, Le Cesne A, Scurr M, Collin F, Pandite L, Marreaud S, Hohenberger P (2012) Cytokine and angiogenic factors associated with efficacy and toxicity of pazopanib in advanced soft tissue sarcoma: an EORTC-STBSG study. Br J Cancer 107: 639-645.

Sternberg CN, Davis ID, Mardiak J, Szczylik C, Lee E, Wagstaff J, Barrios CH, Salman P, Gladkov OA, Kavina A, Zarba JJ, Chen M, Mc Cann L, Pandite L, Roychowdhury DF, Hawkins RE (2010) Pazopanib in locally advanced or metastatic renal cell carcinoma: results of a randomized phase III trial. J Clin Oncol 28: 1061-1068.

Tan AR, Dowlati A, Jones SF, Infante JR, Nishioka J, Fang L, Hodge JP, Gainer SD, Arumugham T, Suttle AB, Dar MM, Lager JJ, Burris 3rd HA (2010) Phase I study of pazopanib in combination with weekly paclitaxel in patients with advanced solid tumors. Oncologist 15: 1253-1261.

Van der Graaf WT, Blay JY, Chawla SP, Kim DW, Bui-Nguyen B, Casali PG, Schöffski P, Aglietta M, Staddon AP, Beppu Y, Le Cesne A, Gelderblom H, Judson IR, Araki N, Ouali M, Marreaud S, Hodge R, Dewji MR, Coens C, Demetri GD, Fletcher CD, Dei Tos AP, Hohenberger P. EORTC Soft Tissue and Bone Sarcoma Group; PALETTE study group (2012) Pazopanib for metastatic soft-tissue sarcoma (PALETTE): a randomised, double blind, placebo-controlled phase 3 trial. Lancet 379: 1879-1886.

Willett CG, Boucher Y, di Tomaso E, Duda DG, Munn LL, Tong RT, Chung DC, Sahani DV, KAalva SP, Kozin SV, Mino M, Cohen KS, Scaddden DT, Hartford AC, Fischman AJ, Clark JW, Ryan DP, Zhi AX, Blaszkowsky LS, Chen HX, Shellito PC, Lauwers GY, Jain RK (2004) Direct evidence that the VEGF-specific antibody bevacizumab has antivascular effects in human rectal cancer. Nat Med 10: 145-147.

This work is published under the standard license to publish agreement. After 12 months the work will become freely available and the license terms will switch to a Creative Commons AttributionNonCommercial-Share Alike 3.0 Unported License. 